\title{
Rocaglamide sensitizes leukemic $T$ cells to activation- induced cell death by differential regulation of CD95L and c-FLIP expression
}

\author{
JY Zhu' ${ }^{1}$, M Giaisi ${ }^{1}$, R Köhler ${ }^{1}$, WW Müller ${ }^{1}$, A Mühleisen ${ }^{1}$, P Proksch ${ }^{2}$, PH Krammer ${ }^{1}$ and M Li-Weber ${ }^{\star, 1}$
}

Drugs with tumor selectivity may have an important benefit in chemotherapies. We have previously shown that Rocaglamide(s), derived from the medicinal plant Aglaia, kills various leukemic cells through the mitochondrial apoptosis pathway with only minor toxicities to normal lymphocytes. Here, we show further that Rocaglamide preferentially promotes activation-induced cell death in malignant T cells by differential regulation of C-FLIP and CD95L expression. Rocaglamide enhances and also prolongs activation-induced JNK activation in malignant $T$ cells leading to downregulation of C-FLIP but upregulation of CD95L expression. We also show that malignant $\mathrm{T}$ cells express a significantly higher amount of Bid - the molecular linker that bridges the receptor-mediated to the mitochondria-mediated apoptosis pathway. Conversely, a substantially lower amount of c-FLIP in response to T-cell stimulation compared to normal T cells is observed. This difference may provide a therapeutic window for cancer treatment. The effect of Rocaglamide on sensitization of activation-induced cell death in malignant $T$ cells was further demonstrated in vivo in a mouse model. Our study demonstrates that Rocaglamide may be a potential anticancer drug that simultaneously targets both C-FLIP and CD95L expressions in tumor cells. This study may also provide a new clue to design a more efficient chemotherapy by using a combination of stimuli that engage the receptor-mediated and the mitochondriamediated death pathway.

Cell Death and Differentiation (2009) 16, 1289-1299; doi:10.1038/cdd.2009.42; published online 17 April 2009

Acute lymphocytic leukemia (ALL) comprises approximately $25 \%$ of acute leukemia cases, of which two-third occur in children. Although the cure rates of childhood ALL are relatively high (approximately $85 \%),{ }^{1}$ the cure rates of adult ALL are poor with only $34-57 \%$ in patients below the age of 30 years, $15-17 \%$ above the age of 50 years and less than $5 \%$ over 60 years of age..$^{2,3}$ Despite many important advances in diagnosis and therapy, the majority of adult ALL patients will die of their disease (American Cancer society, 2006).

Inducing apoptosis in cancer cells is one of the important strategies of anticancer therapy. ${ }^{4,5}$ Apoptotic cell death can be triggered through two different pathways: the extrinsic (receptor-mediated) pathway ${ }^{4,6}$ and the intrinsic (mitochondria-mediated) pathway. ${ }^{5}$ The extrinsic apoptotic pathway is initiated by binding of ligands to specific death receptors on the cell surface which leads to the formation of the death-inducing signaling complex (DISC) containing the FAS-associated death domain adaptor protein (FADD), procaspase-8, and the cellular caspase-8 (FLICE)-like inhibitory protein (c-FLIP). Activation of caspase-8 at the DISC leads to activation of the effector caspase-3, which cleaves a number of target death proteins such as poly(ADP-ribose) polymerase (PARP) leading to apoptosis. ${ }^{6}$ The intrinsic pathway is initiated by various stimuli that directly or indirectly activate the mitochondrial pathway by inducing the release of cytochrome $\mathrm{c}$ and the formation of the apoptosome complex with Apaf- 1 and pro-caspase-9. Caspase- 9 is activated at the apoptosome, and subsequently activates pro-caspase-3. Activation of caspase-8 may induce cleavage of Bid, which links the extrinsic to the intrinsic death pathway by induction of the translocation of the proapoptotic Bcl-2 family proteins Bax and/or Bak to the mitochondrial membrane. ${ }^{6}$

Apoptosis mediated by engagement of CD95 (also known as Fas or Apo-1) and its ligand CD95L plays an important role in immune homeostasis. A known, but still not yet well understood phenomenon is that freshly activated $\mathrm{T}$ cells are resistant to apoptosis, whereas, restimulation of preactivated $\mathrm{T}$ cells through the T-cell receptor (TCR) leads to activationinduced cell death. ${ }^{7}$ Activation-induced cell death in T cells is mediated predominantly by the CD95/CD95L system and is considered to be an important mechanism for contraction of activated $\mathrm{T}$ cells to prevent autoimmunity. ${ }^{8}$ Similar to preactivated $\mathrm{T}$ cells, malignant $\mathrm{T}$ cells are susceptible to activation-induced cell death, probably due to their characteristics similar to proliferating $T$ cells.

In T cells, CD95 is abundantly expressed. ${ }^{7,9}$ In contrast, CD95 L is expressed at an undetectable level in resting $T$ cells, and its expression is rapidly upregulated on T-cell activation.

\footnotetext{
${ }^{1}$ Tumorimmunology Program (D030), German Cancer Research Center (DKFZ), D-69120, Heidelberg, Germany and ${ }^{2}$ Institute of Pharmaceutical Biology and Biotechnology, University of Düsseldorf, Düsseldorf, D-40225 Düsseldorf, Germany

${ }^{*}$ Corresponding author: M Li-Weber, Tumor Immunology Program D030, German Cancer Research Center (DKFZ), Im Neuenheimer Feld 280,69120 Heidelberg, Germany. Tel: 496221 423748; Fax: 496221 411715; E-mail: m.li-weber@dkfz-heidelberg.de

Keywords: anticancer drug; apoptosis; CD95L; c-FLIP; leukemia

Abbreviations: ALL, Acute lymphocytic leukemia; DISC, death-inducing signaling complex; FADD, FAS-associated death domain adaptor protein; C-FLIP, cellular caspase-8 (FLICE)-like inhibitory protein; PARP, poly(ADP-ribose) polymerase; TCR, T-cell receptor

Received 17.12.08; revised 06.3.09; accepted 13.3.09; Edited by V Dixit; published online 17.4.09
} 
Multiple transcription factors such as NF-AT, NF- $\kappa$ B, and AP-1 (Fos/Jun) are involved in transcriptional activation of CD95L expression in activated T cells. ${ }^{10}$ Thus, the rate of CD95-mediated apoptosis is largely determined by the levels of CD95L expression. One major negative regulator of this pathway is the c-FLIP. ${ }^{6,11}$ Three c-FLIP isoforms, c-FLIP , C-FLIP ${ }_{S}$, and $c-$ FLIP $_{R}$, have been identified at the protein level. ${ }^{6} \quad$ C-FLIP $P_{L}$ contains tandem death-effector-domains (DEDs) and a caspase-like domain which lacks amino-acid residues critical for caspase activity. C-FLIP $P_{L}$ inhibits apoptosis by recruitment to and cleavage in the DISC resulting in an inactive caspase- 8 molecule. ${ }^{6,11}$ Recent studies show that C-FLIP $\mathrm{L}_{\mathrm{L}}$ at low expression levels, may also have a proliferative function. ${ }^{6} \mathrm{C}_{\text {-FLIPS }}$ and the recently discovered C-FLIP $P_{R}$ resemble their viral counterparts and have only two DEDs and a short C-terminal part. Recruitment of C-FLIP ${ }_{S}$ and

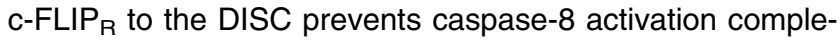
tely. Like CD95L, C-FLIP is also a NF-AT and NF- $\kappa$ B target gene. $^{12-15}$ Thus, CD95L and c-FLIP are coexpressed during T-cell activation. We and others have observed that upregulation of c-FLIP expression during T-cell activation correlates with resistance to CD95/CD95L-mediated apoptosis and rescue of $\mathrm{T}$ cells from activation-induced cell death. ${ }^{16-18}$ To enhance CD95-mediated cell death in malignant $T$ cells c-FLIP would be a promising target.

In recent years, traditional Chinese herbal remedies have gained broad attention as a new source of anticancer drugs. During the past few years, a group of cyclopenta-[b]benzofuran-type compounds, called Rocaglamide (Roc) derived from the traditional Chinese medicinal plants Aglaia, have been found to possess potential anticancer activities in vitro and in vivo in mouse tumor models (for review see reference ${ }^{19}$ ). We have previously shown that Roc(s) selectively kill various human leukemic cells through the mitochondrial pathway without substantial effect on the viability of normal lymphocytes. ${ }^{20}$ The selectivity of Roc was partially explained by its capability to activate the stress-response mitogen-activated protein kinases p38 and JNK in malignant but not normal lymphocytes. ${ }^{20}$ We have also shown that Roc-mediated upregulation of $\mathrm{p} 38$ and JNK activity led to downregulation of NF-AT but upregulation of AP-1 activity in activated leukemic $T$ cells (e.g., Jurkat T cells). ${ }^{21}$ AP-1 plays an essential role in CD95L expression in activated T-cells and also in cells treated with anticancer drugs. ${ }^{22,23}$ As AP-1 has not been described to affect c-FLIP expression, we estimate that Roc may have different effects on the expression levels of CD95L and c-FLIP in malignant T cells. In this study, we show that Roc enhances CD95L-mediated apoptotic cell death in malignant $T$ cells by upregulation of CD95L but downregulation of c-FLIP expression. This effect is further demonstrated in vivo in a mouse lymphoma model. In addition, we show that malignant and normal T cells express distinct levels of c-FLIP and Bid that may provide a therapeutic window to selectively treat malignant $\mathrm{T}$ cells.

\section{Results}

Roc sensitizes malignant T cells toward CD95/CD95Lmediated apoptosis. To investigate whether Roc plays a role in death receptor-mediated apoptosis, two Roc derivatives, Roc-A and Roc-Q (Figure 1a), were investigated in detail in this study. The human leukemic T-cell lines Jurkat, Molt-4, and DND-41 (contains a p53 mutation) and the mouse T lymphoma cell line RMA were stimulated with either $\alpha \mathrm{CD} 3$ or PMA/ionomycin to induce the endogenous CD95L expression in the absence or the presence of different concentrations of Roc. In the absence of Roc, approximately $5-15 \%$ of leukemic $T$ cells underwent activation-induced apoptosis after $24 \mathrm{~h}$ stimulation by PMA/ionomycin or by $\alpha$ CD3. Roc alone also induced about $5-10 \%$ of apoptotic cell death. Noticeably, stimulation of leukemic $T$ cells in the presence of Roc resulted in a dose-dependent increase in apoptotic cell death (Figure 1b). Compared to Jurkat and Molt-4 T cells, DND-41 T cells, which lack p53, were more resistant to Roc- or T-cell stimulation-induced cell death. However, a dramatic increase in apoptotic cell death was observed when DND-41 T cells were treated with the combination of Roc-A and PMA/ionomycin stimulation (Figure 1b). The ability of Roc to increase activation-induced cell death was confirmed by western blot analysis of important proteins involved in the apoptotic pathways. As shown in Figure 1c, T-cell stimulation alone led to only a weak activation of caspase-8, the main caspase involved in the extrinsic pathway, and did not activate caspase-9, the main caspase involved in the intrinsic (mitochondrial) pathway in Jurkat $\mathrm{T}$ cells. Due to the weak activation of caspase-8, T-cell stimulation alone generated very little or almost no detectable amounts of the active caspase-3 cleavage product p17 (although the p19 caspase-3 cleavage products were generated; Figure 1c). Treatment with Roc alone led to activation of caspase- 9 and generation of a detectable amount of the active p17 caspase-3 product and weak PARP cleavage. However, combinations of Roc with T-cell stimulation, in contrast, strongly increased cleavage of caspase-8 (Figure 1c). Enhanced caspase-8 activity correlates with complete cleavage of Bid and consequently enhances cleavage of caspase- 9 and -3 , and PARP (Figure 1c).

To confirm that the Roc-mediated increase in cell death involves the CD95/CD95L system, we carried out an experiment with the anti-CD95L antibody NOK1. In the presence of NOK1, the Roc-mediated increase in T-cell stimulationinduced apoptosis was blocked by at least $50 \%$ indicating that the CD95/CD95L system is involved (Figure 1d). In addition, enhanced apoptotic cell death could be also obtained by treating cells with combinations of Roc with the anti-CD95 antibody ( $\alpha$ APO-1) or with the recombinant LZ-CD95L protein (Figure 1e). Furthermore, Jurkat $T$ cells deficient in the death adaptor molecule FADD (J-FADDdef) did not show an increase in apoptosis by combination treatment (Figure 1f). Such synergistic effect on activationinduced cell death was not seen in combinations with UV irradiation or with anticancer drugs that induce apoptosis mainly through the mitochondrial pathway, for example, daunorubicin, 5-fluorouracil, and cisplatin (Figure 2). These experiments demonstrate that Roc can sensitize death receptor-mediated apoptosis.

Roc downregulates c-FLIP expression. C-FLIP proteins are negative regulators of the extrinsic death pathway at the 


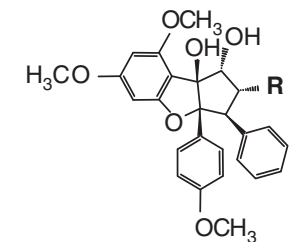

C

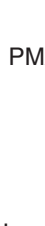

Roc-A: $\mathbf{R}=\mathrm{CON}\left(\mathrm{CH}_{3}\right)_{2} \quad$ Roc-Q: $\mathbf{R}=\mathrm{CONHCH}_{3}$

b

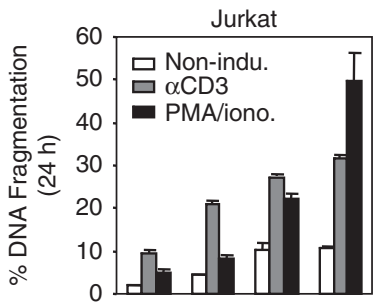

Roc-A (nM): $0 \quad 12.5 \quad 25 \quad 50$

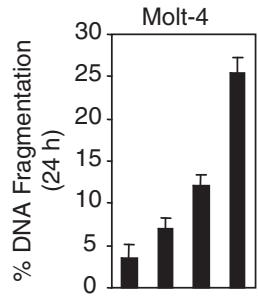

Roc-A (nM): $0 \quad 05050$ PMA/iono: : + - +

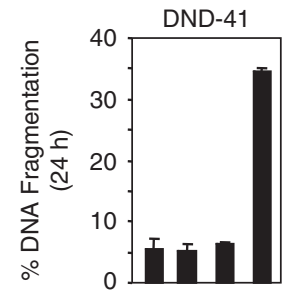

Roc-A (nM): $0 \quad 0 \quad 5050$

PMA/iono: : -+ +

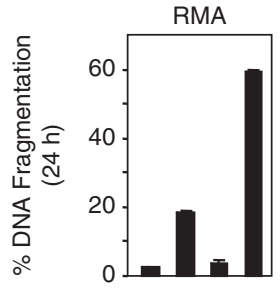

Roc-A (nM): $0 \quad 05050$

PMA/iono.: - + - +

\section{C}

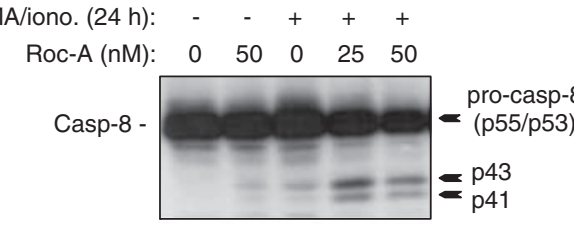

Bid -
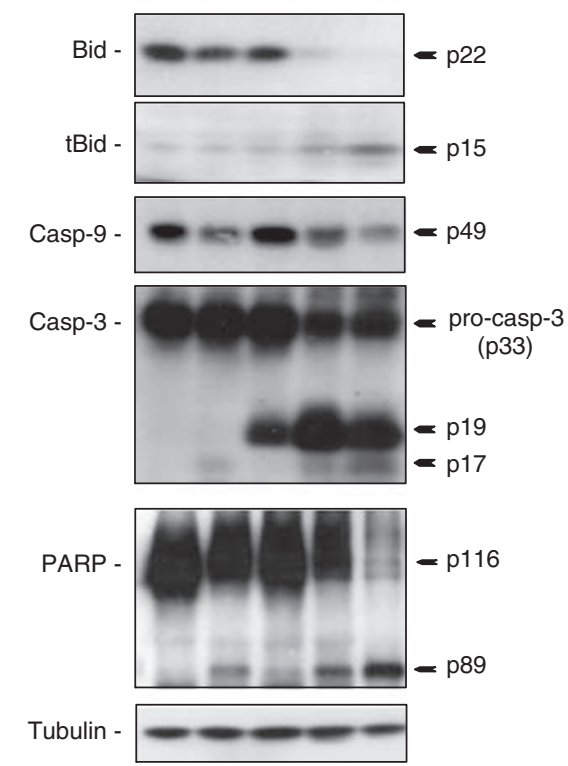

d

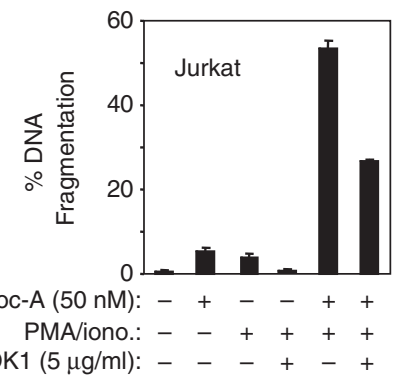

e

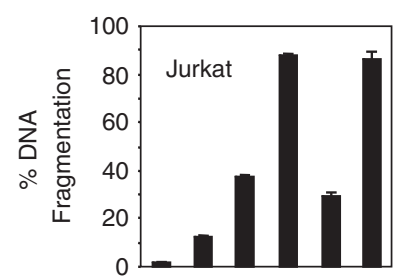

Roc-A (50 nM): -+-+-+ $\alpha \mathrm{APO}-1(0.5 \mathrm{ng} / \mathrm{ml}):-\quad++-$ LZ-CD95L (1:200): - - $\quad$ - + +

f

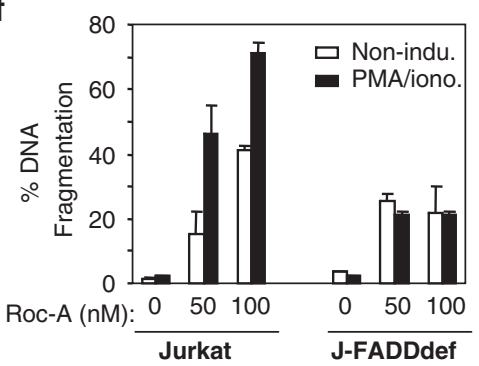

Figure 1 Roc sensitizes malignant T cells toward activation-induced cell death. (a) Chemical structures of Roc (Roc-A and Roc-Q) used in this study. (b) Roc sensitizes for activation-induced apoptosis in leukemic T cells. Human leukemic T-cell lines Jurkat, Molt-4, and DND-41 (which contains a p53 mutation) and a mouse T lymphoma cell line RMA were stimulated with $\alpha \mathrm{CD} 3$ or PMA/ionomycin in the absence or presence of different concentrations of Roc-A as indicated for $24 \mathrm{~h}$. Apoptotic cell death was determined by DNA fragmentation. Results are representative of at least three independent experiments with triplicate assays. (c) Roc-A enhances T-cell -activation-induced cleavage of caspase-8, $-9,-3$, and Bid in leukemic T cells. Jurkat T cells were unstimulated $(-)$ or stimulated with PMA/ionomycin $(+)$ in the absence or the presence of different doses of Roc-A for $24 \mathrm{~h}$. Cell lysates were subjected to western blot with antibodies against caspase-8, $-9,-3$, Bid, PARP, and control antibody against tubulin, sequentially. (d) The $\alpha \mathrm{CD} 95 \mathrm{~L}$ antibody NOK1 downregulates Roc-A-mediated sensitization of cell death. Jurkat T cells were treated with PMA/ionomycin and Roc-A in the absence or the presence of NOK1 for $30 \mathrm{~h}$. Apoptotic cell death was determined by DNA fragmentation. (e) Roc-A increases $\alpha$ APO-1 or LZ-CD95L induced apoptosis. Jurkat T cells were treated with $\alpha A P O-1$ or LZ-CD95L in the absence or the presence of $50 \mathrm{nM}$ Roc-A for $24 \mathrm{~h}$. (f) The death receptor system is required for Roc-mediated elevation of activation-induced cell death. FADD deficient (FADDdef) and parental Jurkat cells were uninduced or induced by PMA/ionomycin in the presence of different doses of Roc-A. All results are representative of two independent experiments in triplicates
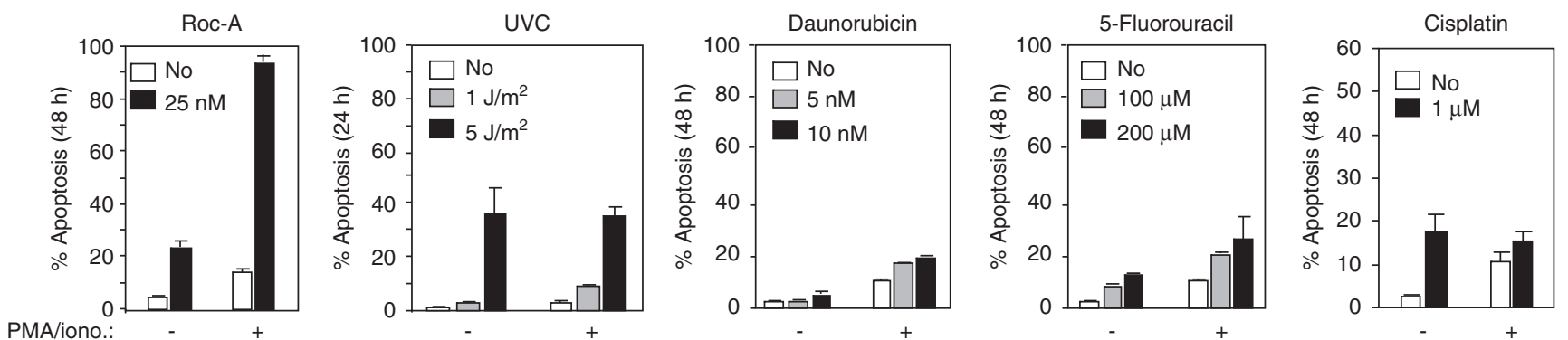

Figure 2 The intrinsic pathway stimuli do not show synergistic effect on activation-induced cell death. Jurkat leukemic T cells were either left unstimulated (No) or stimulated with PMA $(5 \mathrm{ng} / \mathrm{ml})$ and ionomycin $(0.5 \mu \mathrm{M})$ in the absence or presence of different intrinsic pathway stimuli (UV irradiation, anticancer drugs daunorubicin, 5-fluorouracil and cisplatin) as indicated. Roc-A was used as a control. Results are representative of two (for UV) to three (for anticancer drugs) independent experiments

level of the DISC. ${ }^{6,11}$ Therefore, we first examined the expression levels of c-FLIP proteins following Roc treatment. Expression of c-FLIP is transcriptionally controlled by NF- $\kappa \mathrm{B}$ and NF-AT. ${ }^{12,13}$ In contrast to the general view that c-FLIP transcription is controlled predominantly by NF- $\kappa \mathrm{B}$, induction of $\mathrm{c}-\mathrm{FLIP}_{\mathrm{S}}$ in $\mathrm{T}$ cells was recently demonstrated to be 
a

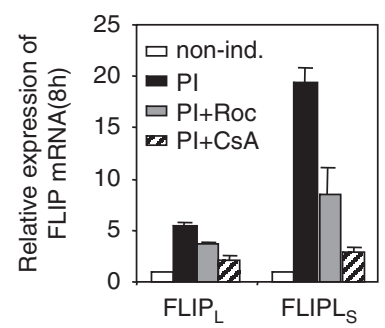

b

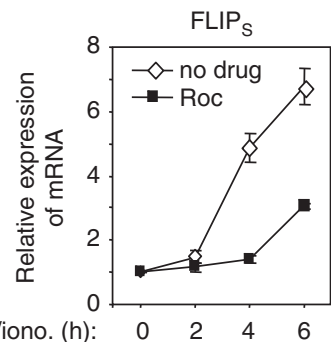

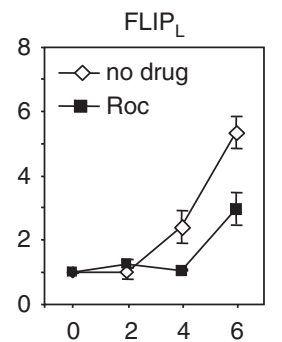

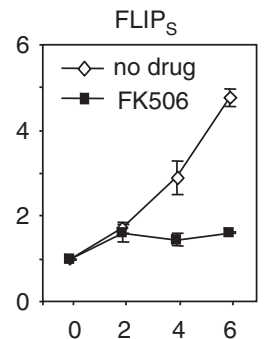

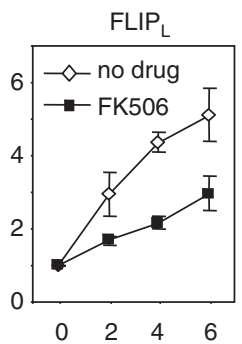

d

C

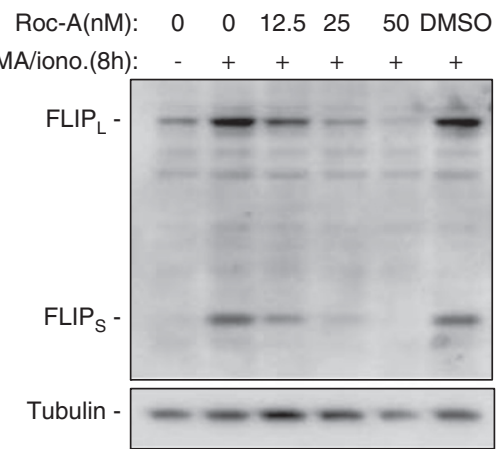

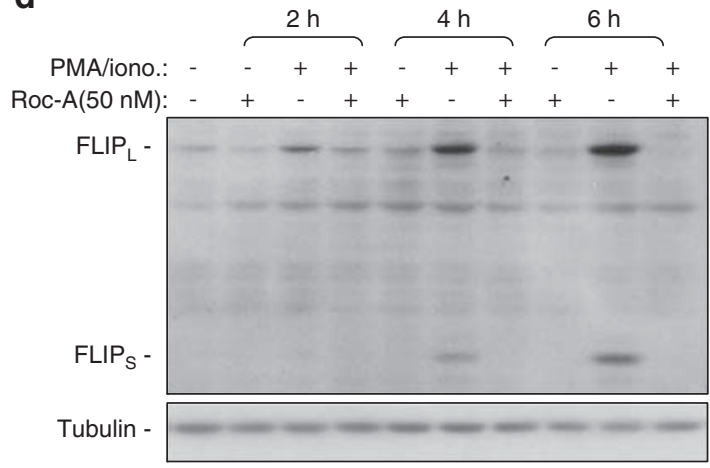

e

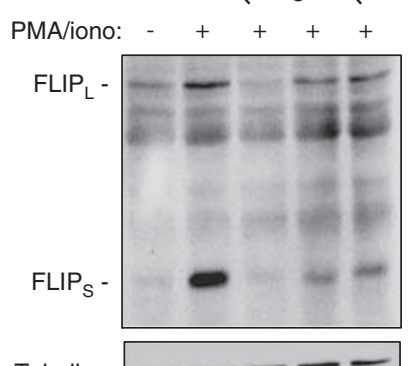

Figure 3 Roc suppresses c-FLIP expression. (a) Roc suppresses T-cell activation-induced c-FLIP mRNA expression. Jurkat T cells were stimulated with PMA/ionomycin in the absence or the presence of Roc-A $(50 \mathrm{nM})$ or CsA $(100 \mathrm{ng} / \mathrm{ml})$ for $8 \mathrm{~h}$. Total mRNA was prepared and analyzed for c-FLIP $\mathrm{F}_{L}$ and c-FLIPS expression by real-time PCR. (b) Kinetic analysis of c-FLIP mRNA expression in the absence or the presence of Roc-A (50 nM) or FK506 (100 ng/ml). Representative data (in triplicate PCRs) from two independent experiments are shown. (c) Roc inhibits c-FLIP protein expression in a dose-dependent manner. Jurkat T cells were stimulated with PMA/ionomycin for $8 \mathrm{~h}$ in the absence or the presence of different amounts of Roc-A. Lysates were analyzed by western blot with an antibody against c-FLIP. One representative experiment of three is shown. (d) Kinetic analysis of the effect of Roc on c-FLIP protein expression. Jurkat T cells were stimulated with PMA/ionomycin in the absence or the presence of $50 \mathrm{nM}$ Roc-A for different time periods as indicated. One representative experiment of two is shown. (e) Effects of CsA and FK506 on cFLIP protein expression. Jurkat T cells were stimulated with PMA/ionomycin in the absence or the presence of CsA $(100 \mathrm{ng} / \mathrm{ml})$ or FK506 $(100 \mathrm{ng} / \mathrm{ml})$ for $6 \mathrm{~h}$. RocA $(50 \mathrm{nM})$ was used as a control. One representative experiment of two is shown

primarily mediated by the NF-AT pathway. ${ }^{13}$ We have previously shown that Roc suppresses NF-AT activity by overactivating the NF-AT kinases p38 and JNK. ${ }^{21}$ Therefore, we predicted that Roc may inhibit c-FLIP expression at the transcriptional level. As expected, in the presence of Roc-A T-cell activation-induced C-FLIP $\mathrm{P}_{\mathrm{L}}$ and $\mathrm{c}$-FLIP $\mathrm{S}$ mRNA expression was downregulated to levels seen when cells were treated with the NF-AT inhibitor CsA (Figure 3a) or FK506 (Figure $3 b$ ). Consistent with the reduced mRNA expression levels, T-cell activation-induced c-FLIP protein expression levels were suppressed by Roc in a dose- (Figure 3c) and time-dependent (Figure 3d) manner. The T-cell stimulationinduced C-FLIP expression was also shown to be downregulated by CsA and FK506 as expected (Figure 3e). We conclude that T-cell stimulation-induced c-FLIP expression can be downregulated by Roc at the transcriptional level.

Roc does not promote activation-induced cell death in normal T cells. We have previously shown that Roc has no or very little toxicity on normal resting peripheral lymphocytes. ${ }^{20}$ To investigate the effect of Roc on activated normal T cells, freshly isolated peripheral blood $T$ cells (day 0 $T$ cells) were stimulated with PMA/ionomycin in the absence or the presence of Roc. Consistent with the previous study, ${ }^{7,20}$ the day 0 (resting) T cells were resistant to PMA/ ionomycin-induced cell death. Roc alone had no toxicity on day $0 \mathrm{~T}$ cells (Figure 4a). Roc also did not increase cell death in combination with PMA/ionomycin stimulation in day $0 \mathrm{~T}$ cells (Figure 4a). Freshly activated T cells are known to be resistant to activation-induced cell death whereas $T$ cells activated for several days in culture become sensitive toward CD95/ CD95L-mediated activation-induced cell death. ${ }^{7}$ Therefore, freshly isolated T cells were stimulated by PHA and further cultured for 6 days (day 6 T cells) and were then restimulated with PMA/ionomycin in the absence or the presence of Roc. Although day $6 \mathrm{~T}$ cells were more susceptible (about 50\% more) to PMA/ionomycin-induced cell death compared to day 0 T cells, Roc did not further enhance PMA/ionomycin-induced cell death in these cells (Figure 4b). Parallel control experiments showed that activation-induced cell death was enhanced by Roc in Jurkat and DND-41 cells (Figure 4c). These data show that Roc preferentially enhances activationinduced cell death in malignant $\mathrm{T}$ cells.

Malignant $\mathrm{T}$ cells express higher levels of Bid but lower levels of c-FLIP than normal T cells. To investigate the reasons that make normal $T$ cells less susceptible to Rocmediated apoptosis than malignant $T$ cells we compared the expression levels of c-FLIP proteins of malignant versus normal $\mathrm{T}$ cells. The experiments showed that following $\mathrm{T}$-cell stimulation, the C-FLIP ${ }_{L}$ and $C-F L I P_{S}$ proteins were increased in both normal and malignant $T$ cells. However, normal day 0 T cells, in contrast to malignant (Jurkat and DND-41) T cells, express much higher levels of $c$-FLIPS on T-cell stimulation 

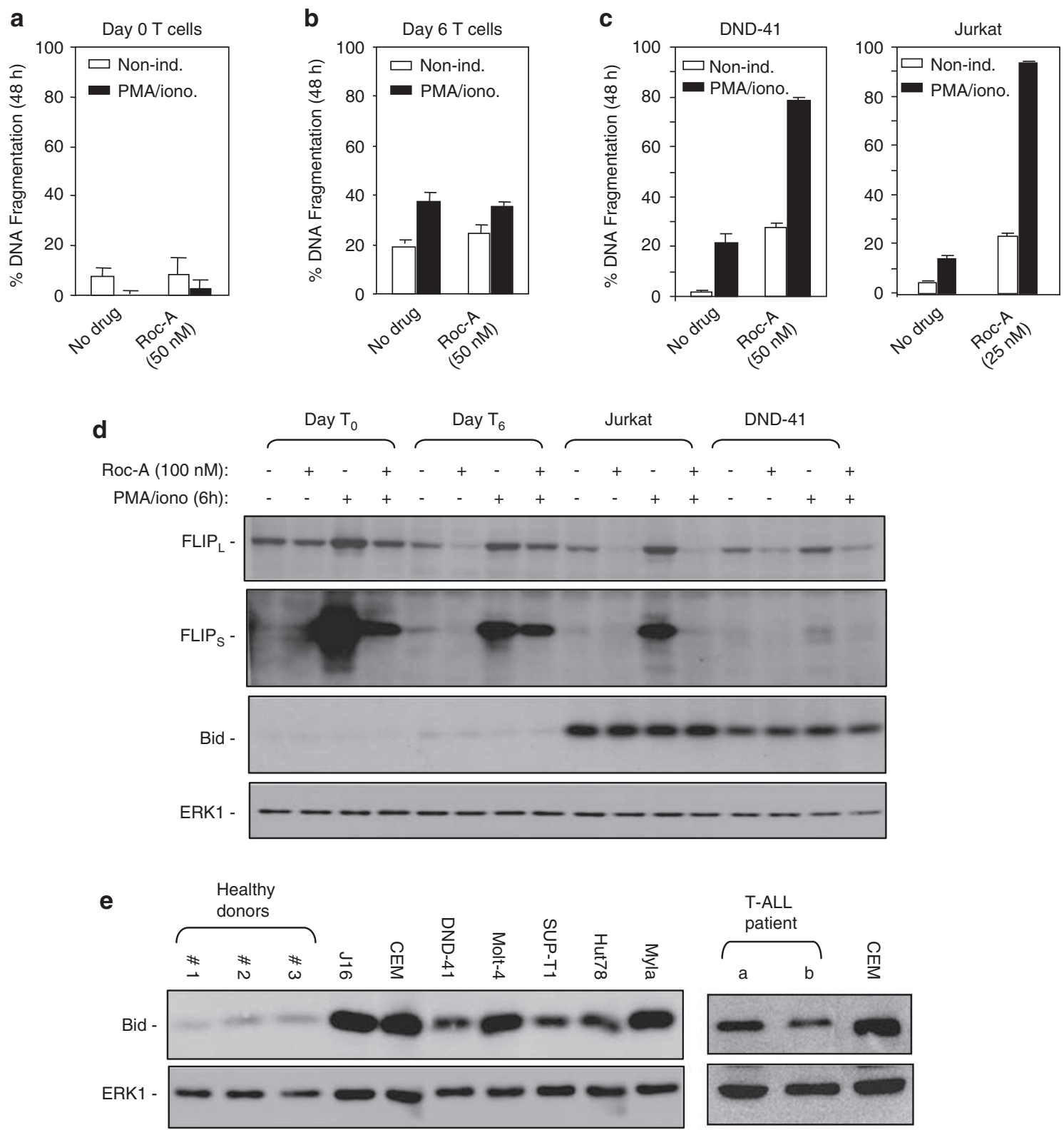

Figure 4 Normal T cells express c-FLIP and Bid at levels different from malignant T cells and are unresponsive to Roc-mediated sensitization of cell death. (a) Roc does not sensitize normal T cells toward activation-induced cell death. Freshly isolated peripheral T cells (day $0 \mathrm{~T}$ cells) were stimulated with PMA/ionomycin in the absence or the presence of Roc-A ( $50 \mathrm{nM}$ ) for $48 \mathrm{~h}$. Apoptosis was determined as percentage of DNA fragmentation. (b) Roc-A $(50 \mathrm{nM})$ also does not substantially sensitize preactivated normal T cells toward activation-induced cell death. Freshly isolated T cells were stimulated by PHA and further cultured for 6 days (day 6 T cells) as described in Materials and Methods. The day $6 \mathrm{~T}$ cells were then restimulated with PMA/ionomycin in the absence or the presence of Roc-A. All data are representative of three independent experiments from three different healthy donors done in triplicates. (c) Jurkat and DND-41 leukemic T cells were treated as in (a) and (b) in parallel as controls. (d) Differences in the expression levels of c-FLIP and Bid in malignant versus normal T cells. Normal day 0 and day 6 T cells and malignant T cell lines Jurkat and DND-41 were activated by PMA/ ionomycin for $6 \mathrm{~h}$ in the absence or the presence of Roc $(100 \mathrm{nM})$. Cell lysates were subjected to western blot analysis with antibodies against c-FLIP and Bid. ERK1 was used as loading control. One representative blot of two independent experiments from two different healthy donors is shown. (e) Differences in the expression levels of Bid in malignant versus normal T cells. Peripheral blood T cells from three healthy donors (donors different from (c)) were compared with different malignant $\mathrm{T}$ cell lines and cells from a T-ALL patient before (A) and after (B) therapy as indicated

(Figure 4d). Roc was shown to completely inhibit c-FLIPS expression in Jurkat and DND-41 T cells but could not completely inhibit c-FLIP $\mathrm{P}_{\mathrm{S}}$ expression in normal day $0 \mathrm{~T}$ cells (Figure 4d). Roc also did not affect the basal level of C-FLIP expression but could reduce the inducible $C-F L I P_{L}$ to its basal level in day $0 \mathrm{~T}$ cells (Figure 4d). Day $6 \mathrm{~T}$ cells, which represent proliferating $T$ cells, were shown to behave similar to Jurkat $T$ cells and expressed c-FLIP at a similar level as Jurkat $T$ cells (Figure $4 d$ ). Roc was shown to reduce the basal as well as the inducible expression levels of C-FLIP but failed to completely inhibit $c-F L I P_{L}$ and $c-F L I P S$ expressions in restimulated day $6 \mathrm{~T}$ cells (Figure $4 \mathrm{~d}$ ). 
Bid is the molecular linker that bridges the extrinsic to the intrinsic pathway of apoptosis. ${ }^{6}$ Surprisingly, we found that malignant $T$ cells express higher levels of Bid than normal $T$ cells (Figure 4d). To investigate this point further, $T$ cells were isolated from additional three healthy donors and were compared to malignant $T$ cell lines established from different patients and from primary leukemic $T$ cells from a T-ALL patient. The experiments showed that Bid is predominantly expressed in malignant $\mathrm{T}$ cells (Figure $4 \mathrm{e}$ ). The above data suggest that the difference in c-FLIP and Bid expressions in normal versus malignant $T$ cells may account for the different sensibilities of these cells toward Roc-mediated sensitization of activation-induced cell death.

\section{Roc enhances CD95L expression in malignant but not in} normal $\mathbf{T}$ cells. We have previously shown that Roc treatment led to activation of p38 and JNK and subsequently enhanced AP-1 activity. ${ }^{21}$ p38 and JNK are NF-AT kinases and, therefore, Roc treatment also results in downregulation of NF-AT activity. ${ }^{21}$ As transcriptional regulation of CD95L expression in T cells is controlled by NF-AT and AP- $1,{ }^{10}$ we asked what role Roc plays in CD95L expression. To answer this question, we examined CD95L mRNA expression levels after T-cell stimulation in the absence or presence of Roc. We observed that in the presence of Roc, CD95L mRNA expression in Jurkat $\mathrm{T}$ cells was downregulated at the early time points (till $4 \mathrm{~h}$ stimulation) but then was dramatically upregulated (Figure 5a, left panel). In contrast, the wellknown NF-AT inhibitor CsA consistently suppressed the expression of CD95L mRNA (Figure 5a, right panel). Interestingly, Roc treatment did not show substantial influence on CD95L mRNA expression in stimulated normal day 0 and day $6 \mathrm{~T}$ cells (Figure $5 \mathrm{~b}$ ). Consistent with the mRNA expression levels, Roc treatment resulted in an increase in surface expression of the CD95L protein primarily in activated Jurkat $T$ cells but not in normal $\mathrm{T}$ cells (Figure $5 \mathrm{c}$ and $\mathrm{d}$ ). Apparently, Roc differentially regulates CD95L expression in malignant versus normal $\mathrm{T}$ cells.

To investigate why Roc did not substantially enhance CD95L expression in normal $T$ cells, we compared the effects of Roc on JNK activity in malignant versus normal

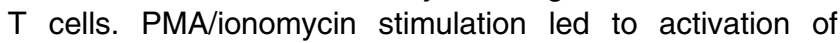
JNK in both Jurkat and normal T cells. Consistent with the previous study, ${ }^{20}$ Roc treatment induces JNK phosphorylation only in malignant cells but not in day 0 (Figure 6a) and day 6 normal $\mathrm{T}$ cells (Supplementary Figure S1). Interestingly, treatment of cells with a combination of Roc and PMA/ ionomycin led to a significant enhancement of JNK activity in malignant cells but had almost no influence in day 0 (Figure 6a) and day 6 normal T cells (Supplementary Figure S1). Kinetic analysis of the effect of Roc on JNK activity in Jurkat $\mathrm{T}$ cells showed that Roc did not only enhance but also significantly prolonged the PMA/ionomycin-induced JNK activity (Figure $6 \mathrm{~b}$ ). Consistent with the previous study, ${ }^{21}$ at the doses used, Roc-A did not prevent PMA/ionomycin-induced $\mathrm{I} \kappa \mathrm{B}$ degradation (Figure $6 \mathrm{~b}$ ). Instead, the levels of $\mathrm{I}_{\kappa} \mathrm{B}$ were even reduced after $8 \mathrm{~h}$ Roc-A treatment, probably due to apoptotic cell death. Transient transfection analysis of an AP-1- and a CD95L-luciferase reporter plasmid into Jurkat $T$ cells confirmed that Roc could enhance the PMA/ionomycin-induced AP-1 and CD95L promoter activity (Figure 6c and d).

In vivo evaluation of the effect of Roc on activationinduced cell death in leukemic $\mathbf{T}$ cells. To explore the potential antitumor effect of Roc in vivo, we carried out an animal study using the mouse T lymphoma cell line RMA as a model system. Similar to human leukemic T cells, Roc-A or Roc- $Q$ enhances PMA/ionomycin- or Con A-stimulationinduced cell death in RMA cells (Figure 1b; Supplementary Figure S2 A). The Roc-enhanced cell death could be inhibited (about 50\%) by the anti-mouse CD95L antibody MFL-3 demonstrating that the CD95/CD95L system was involved (Supplementary Figure S2 B). Roc (A and Q) treatment resulted in downregulation of C-FLIP expression in RMA cells (Supplementary Figure S2 C). AS we had only enough amounts purified Roc- $Q$ available for the animal study, Roc- $Q$ was used in the in vivo experiments. RMA cells were grafted subcutaneously into C57/BL6 mice. One day after xenografting, the mice were randomly separated into four groups (each group contains five mice). One group with no treatment was taken as a control, and the other three groups received Con $\mathrm{A}$, Roc, or a combination of Roc and Con $A$, respectively, three times per week by intraperitoneal injection (i.p.). Two independent experiments were carried out. In the first experiment, RMA $\left(10 \times 10^{5}\right.$ cells)-grafted mice were treated with or without $1 \mathrm{mg} / \mathrm{kg}$ body weight of Roc- $Q$ in the presence or absence of Con $A$ as described in Figure 7a. No obvious toxicity was seen up to a dose of $10 \mathrm{mg} / \mathrm{kg}$ body weight. ${ }^{24}$ Acute toxicity was reported with an $\mathrm{LD}_{50}$ of $>300 \mathrm{mg} / \mathrm{kg}^{25}$ The experiment showed that the control mice developed tumors 7 days after xenografting. No significant changes in tumor size and tumor growth rate were seen in mice treated with only Con A or only Roc-Q. However, delayed tumor growth was seen in mice treated with the combination of Roc- $Q$ and Con A (Figure 7a). To obtain a clearer therapeutic effect, mice were grafted with a reduced amount of tumor cells $\left(2.5 \times 10^{5} \mathrm{RMA}\right.$ cells) and were then treated with an increased dose $(2.5 \mathrm{mg} / \mathrm{kg}$ body weight) of Roc- $Q$ as described in Figure $7 \mathrm{~b}$. In this experiment, the control mice developed tumors after 9 days. Mice that received Con A only showed again no statistically significant change of tumor sizes in comparison to untreated controls ( $P$ value: 0.832 ). Significantly delayed tumor growth was observed in mice, which received Roc- $Q$ only ( $P$ value: 0.021 , compared to no treatment; Figure $7 \mathrm{~b}$ ). Combination treatment led to further inhibition (except for one mouse) of tumor progression ( $P$ value: 0.014 , compared to no treatment; Figure $7 \mathrm{~b}$ ). One mouse in the combination treatment did not show the expected antitumor effect. As all mice that were treated with Roc- $Q$ alone showed reduced tumor sizes and tumor weight, we assume that this exception might be due to an improper delivery of the drugs. No liver toxicity (evaluated by glutamate pyruvate transaminase (GPT) activity; Supplementary Figure S3) and no body weight loss (data not shown) were seen in mice after treatment. 
a

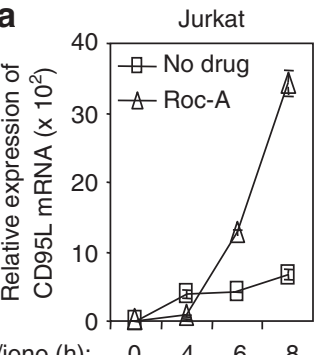

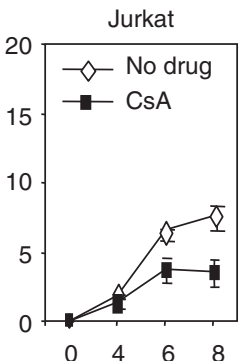

b

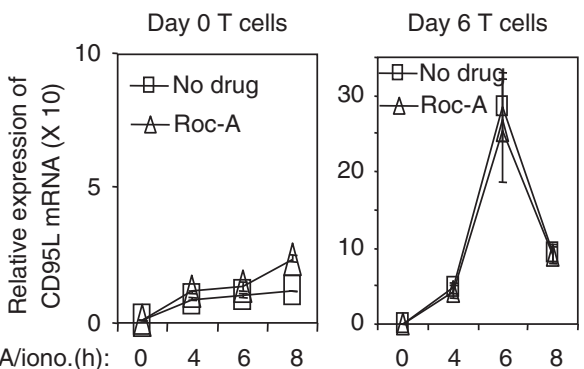

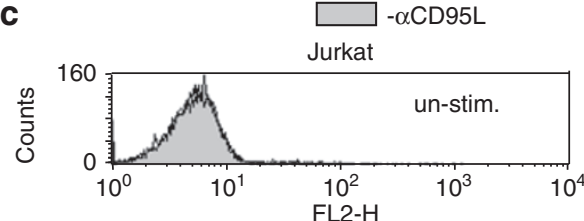
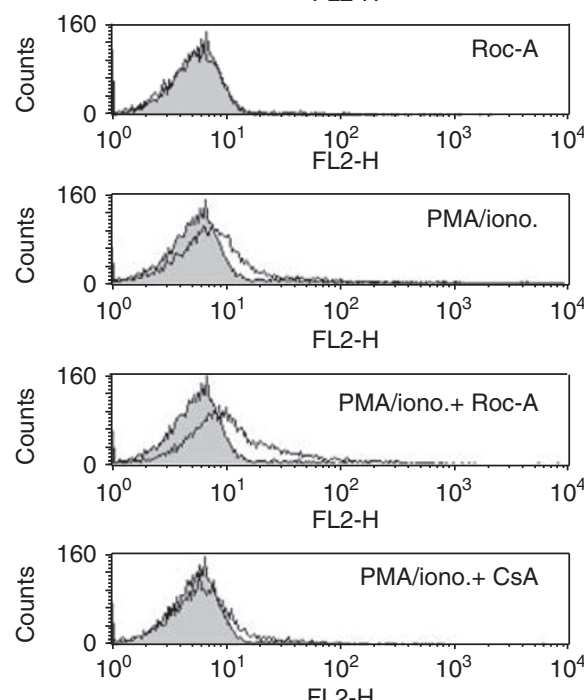

d

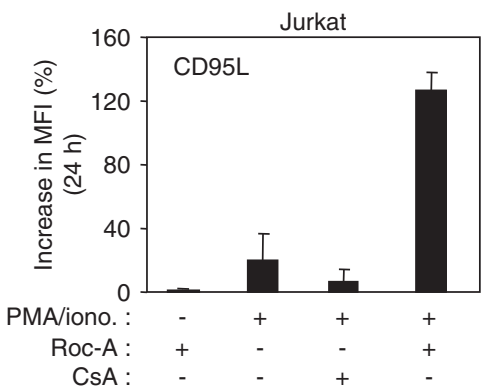

$+\alpha C D 95 \mathrm{~L}$
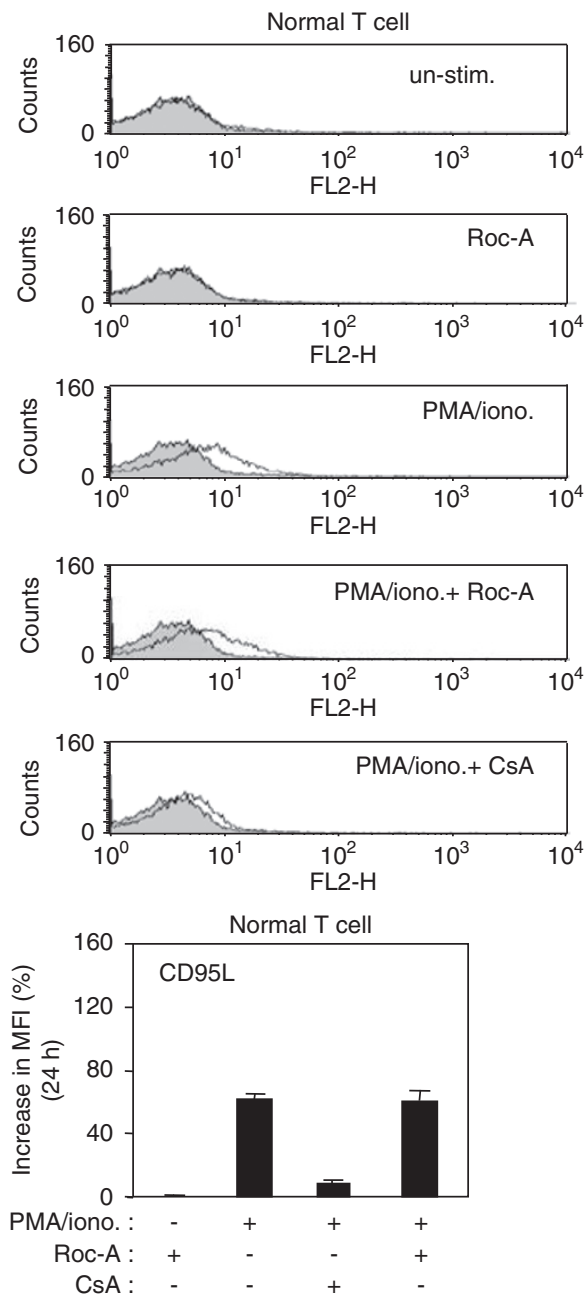

Figure 5 Roc enhances T-cell stimulation-induced CD95L expression. (a) Roc enhances CD95L mRNA expression in malignant T cells. Jurkat T cells were stimulated with PMA/ionomycin in the absence or the presence of Roc-A $(50 \mathrm{nM})$ or CsA $(100 \mathrm{ng} / \mathrm{ml})$ for the indicated time periods. Total mRNA was prepared and analyzed for CD95L expression by real-time PCR. One representative experiment (in triplicate PCRs) of two is shown. (b) Roc does not enhance CD95L mRNA expression in normal T cells. Freshly isolated peripheral blood T cells (day 0 T cells) and T cells further cultured for 6 days after stimulation (day $6 \mathrm{~T}$ cells) were stimulated with PMA/ionomycin in the absence or the presence of Roc-A (50 nM) for the indicated time periods. Total mRNA was analyzed for CD95L expression by real-time PCR. One representative experiment (in triplicate PCRs) of two (different donors) is shown. (c) Roc enhances surface CD95L protein expression in Jurkat but not in normal T cells. A representative FACS analysis of surface CD95L expression is shown following PMA/ionomycin stimulation in the absence or the presence of Roc-A (50 nM). CsA was taken as a positive control for inhibition. (d) The data are presented as a bar chart from triplicate samples. Results are representative of two independent experiments

\section{Discussion}

In this study, we demonstrate that the herbal compound Roc, which is capable to induce mitochondria-mediated apoptosis in leukemia cells, ${ }^{20}$ can also sensitize CD95/CD95L-mediated apoptosis in malignant $\mathrm{T}$ cells by differential regulation of CD95L and c-FLIP expression.

CD95L and c-FLIP are known NF-AT target genes. ${ }^{10,12,13}$ In resting $\mathrm{T}$ cells, NF-AT proteins are phosphorylated and maintained in the cytoplasm. On T-cell stimulation, cytosolic 
a

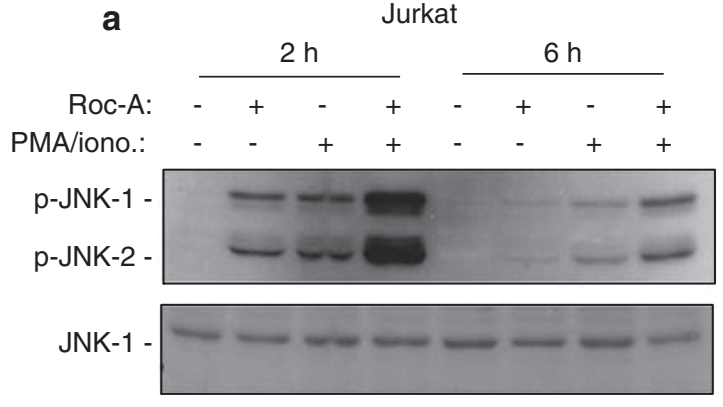

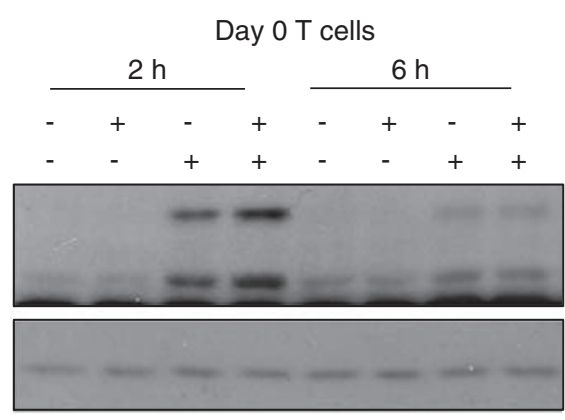

b

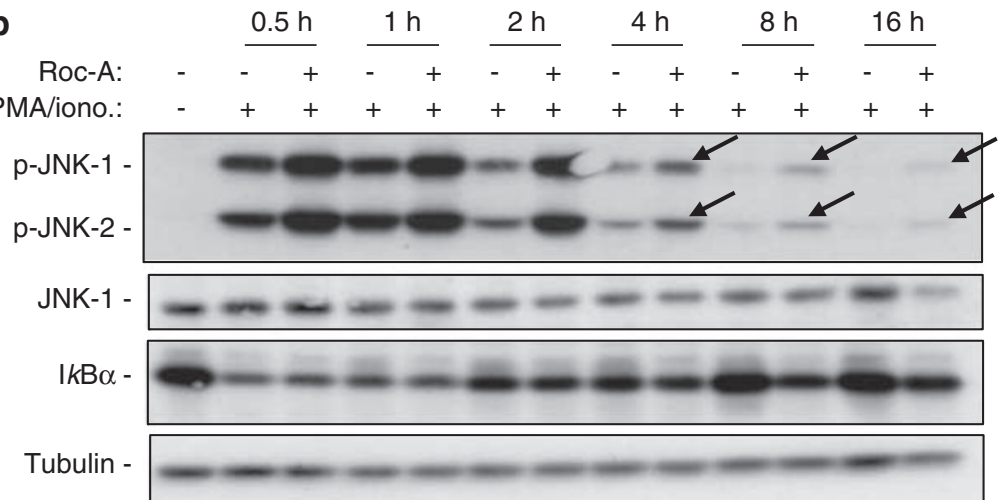

Figure 6 Roc activates JNK and AP-1 activity in malignant but not in normal T cells and enhances CD95L promoter activity in malignant T cells. (a) Roc activates JNK in Jurkat but not in normal T cells. Jurkat and freshly isolated (day 0) peripheral T cells were activated by PMA/ionomycin in the absence or the presence of $50 \mathrm{nM}$ Roc-A for 2 and $6 \mathrm{~h}$. Lysates were subjected to western blot with antibodies against JNK and phosphorylated JNK. Data from normal T cells are representative of two independent experiments with cells from two different healthy donors. (b) Kinetic analysis of effects of Roc on JNK activity and $I_{\kappa} \mathrm{B} \alpha$ degradation. Jurkat T cells were stimulated with PMA/ionomycin in the absence or the presence of Roc-A ( $50 \mathrm{nM})$ for different time periods. Lysates were subjected to western blot with antibodies against phosphorylated JNK ( $\mathrm{p}$-JNK), JNK, $\mid \kappa \mathrm{B} \alpha$, and tubulin. (c and d) Roc enhances T-cell stimulation-induced AP-1 and CD95L promoter activity. Jurkat T cells were transfected with a luciferase reporter construct containing multiple copies of the consensus DNA-binding element of AP-1 or a luciferase reporter construct containing the CD95L promoter. After overnight culture, the cells were split and stimulated with PMA/ionomycin in the absence or presence of Roc-A $(50 \mathrm{nM})$. Luciferase activity was determined $8 \mathrm{~h}$ after stimulation. Data are representative of three independent experiments performed in triplicates

NF-AT proteins are dephosphorylated by the calciumregulated phosphatase calcineurin and shuttled to the nucleus to become engaged in transcription. ${ }^{26}$ This pathway is opposed by extracellular signals, some of which are constitutively active NF-AT kinases, such as casein kinase 1 and glycogen synthase kinase- 3 . The others are inducible kinases, such as p38 and JNK. ${ }^{27,28}$ Both inducible and constitutive kinases rephosphorylate activated NF-AT and cause its nuclear export. The well-known NF-AT inhibitors CsA and FK506 inhibit NF-AT activation by blocking calcineurin function, whereas Roc suppresses NF-AT activity via overactivation of the inducible NF-AT kinases p38 and JNKK. ${ }^{21}$ NF-AT plays an important role in the immediate activation of the CD95L promoter on T-cell stimulation. ${ }^{10,29}$ It is known that NF-AT is an early activated transcription factor, which is rapidly dephosphorylated within minutes of T-cell stimulation and then gradually reverts to its resting (phosphorylated) state $3-5 \mathrm{~h}$ after stimulation. ${ }^{30}$ As shown in our experiments, the expression levels of CD95L mRNA in Jurkat $T$ cells were suppressed by Roc at early activation time points (up to $4 \mathrm{~h}$; Figure 5a). This downregulation may reflect Roc-mediated inhibition of NF-AT. We also show that Roc treatment did not only result in enhanced but also in prolonged JNK activation (Figure 6b). CD95L expression is known to be controlled by the JNK signaling pathway. ${ }^{22,23,31}$ Although the CD95L mRNA expression was suppressed during the early activation time, it was rapidly upregulated after an initial period of stimulation (Figure 5a). Thus, at a later activation phase AP-1 may be the driving force for CD95L expression. In contrast, the calcineurin inhibitor CsA, which does not activate AP-1, suppresses CD95L mRNA expression consistently (Figure 5a) resulting in decreased CD95L protein expression (Figure 5c).

Apparently, c-FLIP mRNA expression in T cells is mainly controlled by NF-AT because Roc does not increase c-FLIP expression at any time point checked (Figure $3 a$ and $b$ ). This result is in agreement with a recent study showing that

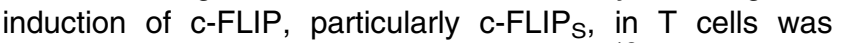
primarily mediated by the NF-AT pathway. ${ }^{13}$ Although the mRNA expression levels show a gradual recovery after $6 \mathrm{~h}$ of activation in the presence of Roc, the c-FLIP protein expression levels were not recovered at this time point (Figure 3d). It has been shown that JNK activation can lead to promotion of protein ubiquitination via phosphorylation of

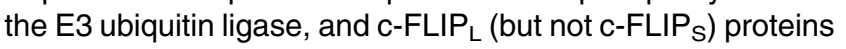
may be eliminated by a JNK-mediated mechanism through the E3 ubiquitin ligase Itch. ${ }^{32,33}$ Thus, Roc-mediated downregulation of $\mathrm{C}-\mathrm{FLIP}_{\mathrm{L}}$ might also involve a proteasomal 


\begin{tabular}{|c|c|c|c|c|c|c|c|}
\hline I & $\downarrow$ & $\downarrow$ & $\downarrow$ & $\downarrow$ & $\downarrow$ & $\downarrow$ & \\
\hline Days: & 0 & 1 & 3 & 6 & $\begin{array}{l}8 \\
4\end{array}$ & $\begin{array}{r}10 \\
4\end{array}$ & 13 \\
\hline
\end{tabular}

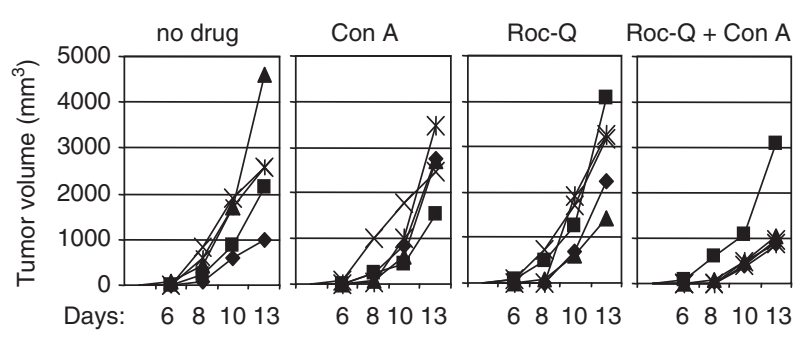

b
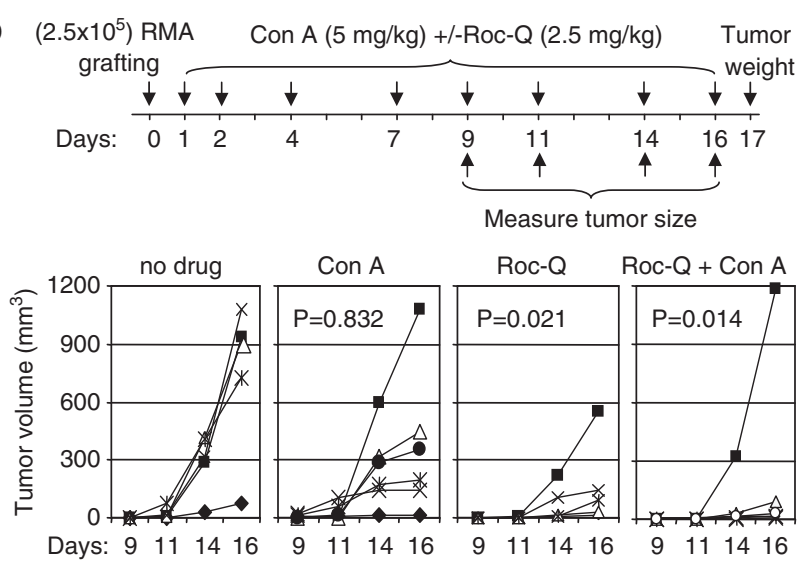

Figure 7 Evaluation of the effect of Roc on activation-induced cell death in malignant T cells in vivo. (a) Twenty C57/BL6 mice were implanted subcutaneously in the right dorsal flank region with RMA $\left(1 \times 10^{6}\right.$ cells). One day after grafting, the mice were randomly separated into four groups and were treated with or without Roc-Q (1 mg/kg), Con A (5 mg/kg), or a combination of both by intraperitoneal injection (i.p.) as indicated in the schematic description. Tumor size was measured 8 days after implantation. (b) The second mouse experiment. Twenty C57/BL6 mice were implanted subcutaneously with $2.5 \times 10^{5}$ RMA cells. One day after grafting, the mice were randomly separated into four groups and were treated with or without Roc-Q (2.5 mg/kg), ConA (5 mg/kg), or a combination of both as indicated. Tumor size was measured after 9 days of implantation. Each symbol represents an individual mouse. $P$ values are obtained by comparing treated mice with untreated control mice

degradation mechanism. This assumption is supported by the observation that in comparison to Roc, CsA, and FK506 treatment led to reduction of $C-F L I P_{L}$ expression only to its basal level (the level before T-cell stimulation), whereas Roc completely inhibited c-FLIP $\mathrm{P}_{\mathrm{L}}$ expression (Figure $3 \mathrm{e}$ ). Downregulation of $C$-FLIP expression then leads to increase of CD95/CD95L-mediated apoptosis.

One of the main obstacles of present chemotherapy in treatment of tumor patients is the toxicity of the drugs. Unfortunately, many existent anticancer drugs also attack proliferating normal cells. We show that Roc did not promote any substantial CD95L expression in activated normal $\mathrm{T}$ cells. This is, in part, due to the fact that Roc does not activate JNK in normal $\mathrm{T}$ cells and, thus, does not enhance T-cell stimulation-induced JNK activity in these cells. We also show that normal T cells (day $0 \mathrm{~T}$ cells) express much higher levels of c-FLIP , and Roc failed to completely inhibit c-FLIP expression in normal $T$ cells (Figure 4d). Furthermore, we found that normal $T$ cells, in contrast to malignant $T$ cells, express significantly less Bid (Figure $4 d$ and e). Bid is the molecular linker bridging extrinsic (as well as various peripheral death pathways) to the central mitochondrial pathway. ${ }^{6}$ Lacking Bid expression indicates that the apoptotic cell death mediated by the extrinsic death pathway cannot be amplified by Bid connecting to the intrinsic death pathway.

The anticancer activities of several Roc derivatives have previously been evaluated in vivo in different tumor models including leukemia models with the mouse p338 lymphoma cell line. ${ }^{34,35}$ In those mouse leukemia models, it was shown that daily injections (i.p.) of $1 \mathrm{mg} / \mathrm{kg}$ of Roc or $2.5 \mathrm{mg} / \mathrm{kg}$ of a Roc derivative Silvestrol resulted in an increase in lifespan of the treated $(\mathrm{T})$ versus the control $(\mathrm{C})$ group by $\mathrm{T} / \mathrm{C}=150 \%$. In our in vivo studies, injection of $2.5 \mathrm{mg} / \mathrm{kg}$ body weight of Roc three times a week in combination with induction of the endogenous CD95L expression by Con A significantly increased the antileukemia effect. Thus, in addition to its direct effect on the intrinsic death pathway, Roc can also sensitize apoptosis mediated by the extrinsic death pathway in vivo.

In summary, we have previously shown that Roc derivatives do not only exert cytostatic but also cytotoxic effects on various human leukemia cell lines and on ALL, CML and AML cells freshly isolated from patients by inducing apoptosis through the mitochondrial pathway. ${ }^{20}$ In this study, we further show that Roc, in addition to its effect on the intrinsic death pathway, can also sensitize apoptosis mediated by the extrinsic death pathway by downregulation of c-FLIP expression. Our study demonstrates that Roc may kill malignant $T$ cells through both intrinsic and extrinsic death pathways in vitro and in vivo. This study may also provide a new clue to design a more efficient chemotherapy against T-cell leukemia by using a combination of stimuli that engage the intrinsic and the extrinsic death pathways.

\section{Materials and Methods}

Cell lines and culture. The mouse T lymphoma cell line RMA, the human leukemic T cell lines DND-41 (contains a p53 mutation), Jurkat (J16), Molt-4, Hut78, CEM, Myla, Sup-T1, and Jurkat (A3) deficient in FADD (J-FADDdef; purchased from American Type Culture Collection (ATCC), Manassas, VA, USA) were cultured in RPMI 1640 medium (GIBCO Laboratories, Grand Island, NY, USA) supplemented with $10 \% \mathrm{FCS}, 50 \mu \mathrm{g} / \mathrm{ml}$ gentamicin (GIBCO), $6 \mathrm{mM}$ HEPES (GIBCO; $1 \mathrm{M}$ solution), and $2 \mathrm{mM}$ L-glutamine (GIBCO; $200 \mathrm{mM}$ solution) at $37^{\circ} \mathrm{C}$ and $5 \% \mathrm{CO}_{2}$. Human $\mathrm{T}$ cells were stimulated with either plate-bound $\alpha \mathrm{CD} 3(\mathrm{OKT} 310 \mu \mathrm{g} / \mathrm{ml})$ or PMA $(5 \mathrm{ng} /$ $\mathrm{ml})$ plus ionomycin $(0.5 \mu \mathrm{M})$. Activation of the mouse RMA cells was carried out with Con A ( $5 \mu \mathrm{g} / \mathrm{ml})$ or PMA plus ionomycin.

Preparation of human $\mathbf{T}$ cells from peripheral blood. Human peripheral T cells were prepared as described previously ${ }^{7}$ and were more than $90 \%$ CD3 positive. For activation, resting T cells (day 0) were cultured at $2 \times 10^{6} \mathrm{cells} / \mathrm{ml}$ with $1 \mu \mathrm{g} / \mathrm{ml} \mathrm{PHA}$ for $16 \mathrm{~h}$ (day 1). Day $1 \mathrm{~T}$ cells were then washed three times and cultured for an additional 5 days in the presence of $25 \mathrm{U} / \mathrm{ml} \mathrm{IL-2} \mathrm{(day} 6$ ).

Determination of apoptosis. Cells were plated in triplicates and treated for the indicated periods of time at $37^{\circ} \mathrm{C}$ with different reagents as described in the figure legends. Rocaglamide derivatives used in this study were isolated from various Aglaia species to at least $98 \%$ purity determined by HPLC as reported previously. ${ }^{21}$ Apoptotic cell death was examined by two parameters: FSC/SSC index of apoptotic-like change in cell size and granularity by FACScan and by analysis of DNA fragmentation. ${ }^{36}$ 
Western blot analysis. $1 \times 10^{7}$ cells were sedimented and lysed for $20 \mathrm{~min}$ in ice-cold lyses buffer $(50 \mathrm{mM}$ Tris- $\mathrm{HCl}, \mathrm{pH} 8.0,120 \mathrm{mM} \mathrm{NaCl}, 1 \% \mathrm{NP}-40,0.5 \%$ Na-Desoxychlat, $0.1 \%$ SDS, 2 mM EDTA, $25 \mathrm{mM} \mathrm{NaF}, 0.2 \mathrm{mM} \mathrm{NaVO}_{4}, 1 \mathrm{mM}$ DTT, and complete protease inhibitor cocktail from Roche). After removing the cell debris by centrifugation at 13,000 r.p.m. for $30 \mathrm{~min}$, equal amounts of proteins were separated on a SDS-PAGE, blotted onto a nitrocellulose membrane (Amersham Biosciences, Little Chalfon, UK) and blocked with $5 \%$ BSA in PBS/Tween (0.05\% Tween-20 in PBS). The following antibodies were used: Caspase- $9 \mathrm{mAb}$ (Santa Cruz Biotechnology, Santa Cruz, CA, USA), caspase-3 polyclonal antibody (Cell Signaling Inc., Beverly, MA, USA), Bid polyclonal antibody recognizes Bid and cleaved Bid (Cell Signaling), anti-JNK1 (C-17; Santa Cruz), anti-phospho-JNK antibody (Cell Signaling), the anti-mouse c-FLIP mAb (Dave-2; Alexis Biochemicals), anti-tubulin (Sigma), anti- $\mid \kappa \mathrm{B} \alpha$ (C21, sc-371; Santa Cruz). The caspase-8 mAb $\mathrm{C} 15$ (mouse IgG2b) recognizes the p18 subunit of caspase-8, the anti-human c-FLIP mAb NF6, the agonistic anti-CD95 mAb and anti-APO-1 were generated in our lab as described previously. ${ }^{37,38}$ For stripping, blots were incubated for $30 \mathrm{~min}$ in a buffer containing $62.5 \mathrm{mM}$ Tris/ $\mathrm{HCl}, \mathrm{pH} 6.8,2 \% \mathrm{SDS}$, and $100 \mathrm{mM} \beta$-mercaptoethanol at $56^{\circ} \mathrm{C}$. The blots were washed six times for $10 \mathrm{~min}$ in $\mathrm{PBS} /$ Tween and blocked again in $5 \% \mathrm{BSA}$.

Quantitative real-time PCR. The primers and fluorescent-labeled probes for TaqMan quantitative real-time $\mathrm{PCR}$ for $\mathrm{CD} 95 \mathrm{~L}$ are $\mathrm{CD} 95 \mathrm{~L}$ forward primer $5^{\prime}(508)$ AAAGTGGCCCATTTAACAGGC-3', reverse primer $5^{\prime}(599)$-AAAGCAGGACAAT TCCATAGGTG-3' ${ }^{\prime}$, and probe $5^{\prime}(542)$-TCCAACTCAAGGTCCATGCCTCTGG- $3^{\prime}$; for $c-F L I P_{L}, c-F L I P_{S}$, and $\beta$-actin (used as an internal standard) were described previously. ${ }^{39}$ Briefly, PCR was performed in a $12.5 \mu$ reaction mixture (PCR kit from Eurogentech, Belgium) that contained $0.08 \mu \mathrm{g}$ of reverse transcribed cDNA and proper amounts of primers and probe. For each sample, three PCR reactions were performed. The resulting relative increase in reporter fluorescent dye emission was monitored by the TaqMan-system (GeneAmp 5700 sequence detection system and software; Perkin Elmer, Foster City, CA, USA). The mRNA levels of the target genes, relative to $\beta$-actin, was calculated using the formula: Relative mRNA expression $=2^{-(\Delta \mathrm{Ct} \text { of tested genes }-\Delta \mathrm{Ct} \text { of } \beta \text {-actin })}$ where $\mathrm{Ct}$ is on the threshold cycle value.

Plasmid constructs and transient transfections. The luciferase reporter constructs containing multiple copies of the AP-1 binding site from SV40 enhancer (CGGTTGCTGACTAATTG) and the CD95L promoter (-860) sequence were described previously. ${ }^{29}$ Jurkat T cells were transfected by electroporation as previously described. ${ }^{29}$ After overnight recovery, the cells were divided and stimulated with PMA $(5 \mathrm{ng} / \mathrm{ml})$ and ionomycin $(0.5 \mu \mathrm{M})$ in the absence or presence of Roc for $8 \mathrm{~h}$. Luciferase activity was determined in $10 \mu \mathrm{l}$ of cell extract using the luciferase assay substrate (Promega Corp., Mannheim, Germany) with a Duolumat LB9507 luminometer (Berthold, Bad Wildbad, Germany).

In vivo mouse studies. C57/BL6 mice were implanted subcutaneously in the right dorsal flank region with the mouse lymphoma cell RMA. One day after grafting, Roc-Q (dissolved in DMSO and diluted in olive oil) was administered by intraperitoneal injection (i.p.) as indicated in the figure legend. The control group was treated in an analogous manner with the vehicle. The tumor size was measured with a micrometer caliper at the indicated times and the tumor volume $(V)$ was calculated by the formula $V=\left(a^{2} \times b\right) / 2$, where ' $a$ ' is the width and ' $b$ ' is the length in $\mathrm{mm} .{ }^{40}$ All protocols using and maintaining animals were approved by the German Animal Protection Authority (Office Regierungspräsidium Karlsruhe). Treated and control animals were compared for differences in tumor growth after end of treatment using the nonparametric method in a one-sided statistical test at the significance level of $0.05{ }^{40}$

Quantitative determination of glutamate pyruvate transaminase. To examine the liver toxicity of Rocaglamide, the activities of GPT were determined from the heparinized blood of mice by test strips Reflotron GPT as described by the instruction of the manufacture (Roche 10745138). The normal levels of GPT activity should be below $50 \mathrm{U} / \mathrm{l}$.

Acknowledgements. We thank Dr. W Rittgen for the biostatistics analysis. We also thank Dr. K-M Debatin and Dr. L-H Meyer (Universitätsklinikum Ulm, Klinik für Kinder- und Jugendmedizin, UIm) for kindly providing patient T-ALL cells. This work was supported by the Deutsche Krebshilfe in Germany and by the Alliance for Immunotherapy of the Helmholtz Society.
1. Pui CH, Evans WE. Acute lymphoblastic leukemia. N Engl J Med 1998; 339: 605-615.

2. Annino L, Vegna ML, Camera A, Specchia G, Visani G, Fioritoni G et al. Treatment of adult acute lymphoblastic leukemia (ALL): long-term follow-up of the GIMEMA ALL 0288 randomized study. Blood 2002; 99: 863-871.

3. Rowe JM, Buck G, Burnett AK, Chopra R, Wiernik PH, Richards SM et al. Induction therapy for adults with acute lymphoblastic leukemia: results of more than 1500 patients from the international ALL trial: MRC UKALL XI/ECOG E2993. Blood 2005; 106: 3760-3767.

4. Debatin KM, Krammer PH. Death receptors in chemotherapy and cancer. Oncogene 2004; 23: $2950-2966$

5. Galluzzi L, Larochette N, Zamzami N, Kroemer G. Mitochondria as therapeutic targets for cancer chemotherapy. Oncogene 2006; 25: 4812-4830.

6. Krammer PH, Arnold R, Lavrik I. Life and death in peripheral T cells. Nat Rev Immunol 2007; 7: 532-542.

7. Klas C, Debatin KM, Jonker RR, Krammer PH. Activation interferes with the APO-1 pathway in mature human T cells. Int Immunol 1993; 5: 625-630.

8. Brenner D, Krammer PH, Arnold R. Concepts of activated T cell death. Crit Rev Oncol Hematol 2008; 66: 52-64.

9. Ogasawara J, Suda T, Nagata S. Selective apoptosis of CD4+CD8+ thymocytes by the anti-Fas antibody. J Exp Med 1995; 181: 485-491.

10. Li-Weber M, Krammer PH. Function and Regulation of CD95 (APO-1/Fas) Ligand in the Immune System. Semin Immunol 2003: 15: 145-157.

11. Budd RC, Yeh WC, Tschopp J. cFLIP regulation of lymphocyte activation and development. Nat Rev Immunol 2006; 6: 196-204.

12. Zaichuk TA, Shroff EH, Emmanuel R, Filleur S, Nelius T, Volpert OV. Nuclear factor of activated T cells balances angiogenesis activation and inhibition. J Exp Med 2004; 199: 1513-1522.

13. Ueffing N, Schuster M, Keil E, Schulze-Osthoff K, Schmitz I. Up-regulation of c-FLIP short by NFAT contributes to apoptosis resistance of short-term activated T cells. Blood 2008; 112: $690-698$.

14. Kreuz $\mathrm{S}$, Siegmund $\mathrm{D}$, Scheurich $\mathrm{P}$, Wajant $\mathrm{H}$. NF-kappaB inducers upregulate $\mathrm{CFLIP}$, a cycloheximide-sensitive inhibitor of death receptor signaling. Mol Cell Biol 2001; 21 3964-3973.

15. Micheau O, Lens S, Gaide O, Alevizopoulos K, Tschopp J. NF-kappaB signals induce the expression of c-FLIP. Mol Cell Biol 2001; 21: 5299-5305.

16. Kirchhoff S, Muller WW, Krueger A, Schmitz I, Krammer PH. TCR-mediated up-regulation of c-FLIPshort correlates with resistance toward CD95-mediated apoptosis by blocking death-inducing signaling complex activity. J Immunol 2000; 165: 6293-6300.

17. Schmitz I, Weyd H, Krueger A, Baumann S, Fas SC, Krammer PH et al. Resistance of short term activated $T$ cells to CD95-mediated apoptosis correlates with de novo protein synthesis of c-FLIPshort. J Immunol 2004; 172: 2194-2200.

18. Zhang N, Hopkins K, He YW. C-FLIP protects mature T lymphocytes from TCR-mediated killing. J Immunol 2008; 181: 5368-5373.

19. Kim S, Salim AA, Swanson SM, Kinghorn AD. Potential of cyclopenta[b]benzofurans from Aglaia species in cancer chemotherapy. Anticancer Agents Med Chem 2006; 6: 319-345.

20. Zhu JY, Lavrik IN, Mahlknecht U, Giaisi M, Proksch P, Krammer PH et al. The tradition Chinese herbal compound rocaglamide preferentially induces apoptosis in leukemia cells by modulation of MAPK activities. Int J Cancer 2007; 121: 1839-1846.

21. Proksch P, Giaisi M, Treiber MK, Palfi K, Merling A, Spring H et al. Rocaglamide derivatives are immunosuppressive phytochemicals that target NF-AT activation in T cells. J Immunol 2005; 174: 7075-7084.

22. Eichhorst ST, Muller M, Li-Weber M, Schulze-Bergkamen H, Angel P, Krammer PH. A novel AP-1 element in the CD95 Ligand promoter is required for induction of apoptosis in hepatocellular carcinoma cells upon treatment with anticancer drugs. Mol Cell Biol 2000; 20: 7826-7837.

23. Baumann S, Hess J, Eichhorst ST, Krueger A, Angel P, Krammer PH et al. An unexpected role for FosB in activation-induced cell death of T cells. Oncogene 2003; 22: 1333-1339.

24. Lee SK, Cui B, Mehta RR, Kinghorn AD, Pezzuto JM. Cytostatic mechanism and antitumor potential of novel $1 \mathrm{H}$-cyclopenta[b]benzofuran lignans isolated from Aglaia elliptica. Chem Biol Interact 1998; 115: 215-218.

25. Umezawa K, Yamamoto T, Koyano T. Suppressant for cancer gene function. Japanese Patent JP 9067375(A) 1997.

26. Rao A, Luo C, Hogan PG. Transcription factors of the NFAT family: regulation and function. Annu Rev Immunol 1997; 15: 707-747.

27. Gómez del Arco P, Martínez-Martínez S, Maldonado JL, Ortega-Pérez I, Redondo JM. A role for the p38 MAP kinase pathway in the nuclear shuttling of NFATp. J Biol Chem 2000; 275: 13872-13878.

28. Chow CW, Dong D, Flavell RA, Davis RJ. c-Jun NH(2)-terminal kinase inhibits targeting of the protein phosphatase calcineurin to NFATc1. Mol Cell Biol 2000; 20: 5227-5234.

29. Li-Weber M, Laur O, Krammer PH. Novel Egr/NF-AT composite sites mediate activation of the CD95 (APO-1/Fas) ligand promoter in response to T-cell stimulation. Eur J Immunol 1999; 29: 3017-3027.

30. Loh C, Carew JA, Kim J, Hogan PG, Rao A. T-cell receptor stimulation elicits an early phase of activation and a later phase of deactivation of the transcription factor NFAT1. Mol Cell Biol 1996; 16: 3945-3954.

31. Kasibhatla S, Brunner T, Genestier L, Echeverri F. Mahboubi A and Green DR, DNA damaging agents induce expression of Fas ligand and subsequent apop- 
tosis in T lymphocytes via the activation of NF-kappa B and AP-1. Mol Cell 1998; 1 : 543-551.

32. Gao M, Karin M. Regulating the regulators: control of protein ubiquitination and ubiquitinlike modifications by extracellular stimuli. Mol Cell 2005; 19: 581-593.

33. Chang L, Kamata H, Solinas G, Luo JL, Maeda S, Venuprasad K et al. The E3 ubiquitin ligase itch couples JNK activation to TNFalpha-induced cell death by inducing c-FLIP(L) turnover. Cell 2006; 124: 601-613.

34. King ML, Chiang CC, Ling HC, Fujita E, Ochiai M, McPhail AT. X-ray crystal structure of rocaglamide, a novel antileukemic $1 \mathrm{H}$-cyclopenta[b]benzofuran from Aglaia elliptifolia. J Chem Soc, Chem Commun 1982: 1150-1151.

35. Hwang BY, Su BN, Chai H, Mi Q, Kardono LB, Afriastini JJ et al. Silvestrol and episilvestrol, potential anticancer rocaglate derivatives from Aglaia silvestris. J Org Chem 2004; 69 : 3350-3358
36. Vermes I, Haanen C, Reutelingsperger C. Flow cytometry of apoptotic cell death $\mathrm{J}$ Immunol Methods 2000; 243: 167-190.

37. Scaffidi C, Medema JP, Krammer PH, Peter ME. FLICE is predominantly expressed as two functionally active isoforms, caspase-8/a and caspase-8/b. J Biol Chem 1997; 272: 26953-26958.

38. Trauth BC, Klas C, Peters AM, Matzku S, Möller $\mathrm{P}$, Falk $W$ et al. Monoclonal antibody-mediated tumor regression by induction of apoptosis. Science 1989; 245 : 301-305.

39. Krueger A, Fas SC, Giaisi M, Bleumink M, Merling A, Stumpf C et al. HTLV-1 Tax protects against CD95-mediated apoptosis by induction of the cellular FLICE-inhibitory protein (c-FLIP). Blood 2006; 107: 3933-3939.

40. Mattern J, Bak M, Hahn EW, Volm M. Human tumor xenografts as model for drug testing. Cancer Metastasis Rev 1998; 7: 263-284.

Supplementary Information accompanies the paper on Cell Death and Differentiation website (http://www.nature.com/cdd) 